\title{
Entorno Virtual de Enseñanza y Aprendizaje Aplicado como Recurso Instruccional Complementario en la Enseñanza de Neuroanatomía:Aspectos Poblacionales, Didácticos y Psicopedagógicos
}

\author{
Virtual Teaching and Learning Applied as Supplementary Instructional Resources \\ in Teaching Neuroanatomy: Population, Educational and Psychopedagogic Aspects
}

Elba B. Tornese; Claudia G. Dogliotti; Martín J. Mazzoglio y Nabar; Rubén D. Algieri; Andrea Gazzotti; Humberto N. Jiménez Villarruel; Lorena M. Rey \& Ananquel Gómez

TORNESE, E. B.; DOGLIOTTI, C. G.; MAZZOGLIO Y NABAR, M. J.; ALGIERI, R. D.; GAZZOTTI, A.; JIMÉNEZ VILLARRUEL, H. N.; REY, L. M. \& GÓMEZ, A. Entorno virtual de enseñanza y aprendizaje aplicado como recurso instruccional complementario en la enseñanza de neuroanatomía: aspectos poblacionales, didácticos y psicopedagógicos. Int. J. Morphol., 29(4):11301135, 2011.

RESUMEN: Las nuevas tecnologías de la información y la comunicación están cambiando la sociedad e influyen fuertemente en la educación, creando escenarios nuevos y valiosos tanto para la enseñanza como el aprendizaje. Estos entornos virtuales de enseñanza y aprendizaje (EVEA) son plataformas informáticas pensadas para facilitar la comunicación pedagógica entre los participantes en un marco de proceso educativo. El objetivo es presentar resultados de adherencia, rendimiento y utilidad didáctica del EVEA, especificando características, cuestiones poblacionales y psicopedagógicas subyacentes, al aplicarlo en Neuroanatomía. Diseñamos un EVEA mediante la utilización de Moodle ${ }^{\circledR}$ con actividades de consulta, tarea, foros de aprendizaje, wiki, hipertexto y cuestionario. Se implementó en 3 comisiones de la cursada 2010 de forma 1 obligatoria y 2 no obligatoria y se evaluaron parámetros para conocer la eficacia del recurso pedagógico implementado como complementario con contenidos curriculares de los trabajos prácticos. Los resultados fueron evaluados con herramientas de estadística descriptiva. El 75\% de los alumnos utilizó el EVEA, la frecuencia de acceso mayor fue noche (20-24 hs). Detectamos dificultad en el uso diario de esta herramienta pese a su fácil acceso. La mitad ingresó 2 veces/semana, sin prevalencia en cuanto al día, mayormente 1 vez/día, desde el hogar, y permaneció logueado en el sitio entre 30 y 70 minutos (60\%). Determinamos correlación entre resultados del parcial insatisfactorio y menor uso del recurso (R2>0,78). Objetivamos beneficios en el empleo de EVEA en la rotación Neuroanatomía. Dicho recurso genera la construcción del proceso de enseñanza y aprendizaje mediante la reelaboración del contenido mediada por la estructura cognitiva subjetiva del alumno.

PALABRAS CLAVE: Neuroanatomía; Pedagogía; EVEA.

\section{INTRODUCCIÓN}

Las nuevas tecnologías de la información y la comunicación (TIC) están cambiando la sociedad e influyen fuertemente en la educación, creando escenarios nuevos y valiosos tanto para la enseñanza como el aprendizaje (Algieri et al., 2009; Tornese et al., 2010). Los distintos niveles y modalidades de la educación (pregrado, grado, posgrado, presencial, semipresencial o a distancia) deben adaptarse y adaptar las TICs como una herramienta en sus actividades (Algieri et al., 2009; Algieri et al., 2008; Tornese et al.). Consideramos, en sintonía con lo postulado por la Prof. Lic. Edith Litwin, que "no es la tecnología la que nos permite producir los cambios, sino nuestra decisión de imaginar con y a través de ella" (Litwin, 2005).
Los entornos virtuales de enseñanza y aprendizaje (EVEA) son un tipo de e-learning basado en plataformas informáticas diseñadas para facilitar la comunicación pedagógica entre los participantes en un marco de proceso educativo. Son un tipo de TIC que permite almacenar, editar, transmitir, recibir y enviar informaciones en mensajes de texto y audiovisuales dentro de un espacio que favorece la multidireccionalidad entre participantes.

El objetivo del presente trabajo es presentar los resultados de adherencia, rendimiento y utilidad didáctica de la implementación de un EVEA creado por nuestro grupo de trabajo con foco en problemáticas poblacionales y 
TORNESE, E. B.; DOGLIOTTI, C. G.; MAZZOGLIO Y NABAR, M. J.; ALGIERI, R. D.; GAZZOTTI, A.; JIMÉNEZ VILLARRUEL, H. N.; REY, L. M. \& GÓMEZ, A. Entorno virtual de enseñanza y aprendizaje aplicado como recurso instruccional complementario en la enseñanza de neuroanatomía: aspectos poblacionales, didácticos y psicopedagógicos. Int. J. Morphol., 29(4):1130-1135, 2011.

psicopedagógicas subyacentes, al aplicar nuevos instrumentos de enseñanza a la Neuroanatomía Básica.

\section{MATERIAL Y MÉTODO}

Diseñamos un EVEA mediante la utilización del software Moodle ${ }^{\circledR}$ y se implementó en 3 comisiones (C3, C4 y C7) del año 2010 correspondientes a la cursada de Anatomía de la $3^{\circ}$ Cátedra de Anatomía Humana de la Facultad de Medicina de la Universidad de Buenos Aires. La utilización del EVEA fue opcional en 2 comisiones y se evaluaron parámetros predeterminados para conocer la eficacia del recurso pedagógico como complementario con los contenidos curriculares de los trabajos prácticos (TP). Las características poblacionales de los participantes se exhiben en la Tabla I.

El EVEA incluyó: actividades fuera de línea, tareas de texto en línea, foros de aprendizaje, glosarios, espacio de consultas y wikis. Se proporcionó a los participantes en cada TP el material en forma de resolución de casos clínicos, aplicación de estudios por imágenes, mapas conceptuales, implementación de terminología anatómica y clases de profundización de conocimientos en formato pdf.

Se registraron calificaciones conceptuales en los TP, de parciales y recuperatorios y se valoró la utilidad del recurso pedagógico en función de la cantidad de inscriptos, horas y rango horario de ingreso, frecuencia de acceso, beneficio para el proceso de enseñanza de los foros de discusión y de los materiales disponibles.

Los resultados obtenidos fueron evaluados con pruebas estadísticas y el procedimiento se realizó bajo el marco de reparos éticos (requerimiento del consentimiento informado para investigaciones por medios informáticos, cum- plimiento de requisitos estipulados por las GCP, Disposición ANMAT 6677/10 y adhesión a principios éticos con origen en la Declaración de Helsinski).

\section{RESULTADOS}

El 75\% de los alumnos inscriptos utilizó el EVEA (128 alumnos de forma obligatoria y 164 de 217 de forma optativa). En relación con el perfil poblacional, en el grupo perteneciente a comisiones con uso optativo (CUOP) el 93,15\% tenía entre 19 y 28 años con el $62,33 \%$ de sexo femenino, el $63,01 \%$ no trabajaba y el $89,73 \%$ no tenía experiencia previa en e-learnining. En la comisión con uso obligatorio (CUOB) el 97,66\% tenía entre 19 y 28 años con el $75 \%$ de sexo femenino, el 78,91\% no trabajaba y el $83,59 \%$ no tenía experiencia previa en elearnining.

En relación con los parámetros de ingreso y uso del EVEA, el 59,38\% de los alumnos CUOB refiere descargar al ordenador e imprimir los archivos, mientras que el 60,27\% de CUOP algunos los guarda y otros los ve en línea. Registramos que el 55,09\% ingresó una vez por día en ambos grupos, pero en los CUOP se registró mayor ingreso por semana. Los alumnos de CUOB prefirieron ingresar desde su domicilio $(82,03 \%)$, durante la noche $(50,78 \%)$ o en la tarde $(30,47 \%)$, con un tiempo promedio de logueo en el sistema de 30 a 60 minutos $(39,84 \%)$; mientras en los CUOP prevaleció el ingreso desde el hogar $u$ otros lugares $(63,70 \%, 47,26 \%)$, durante la noche $(69,18 \%)$ u horario vespertino $(13,01 \%)$, con un tiempo de logueo en la plataforma de 30 a 60 minutos $(52,05 \%)$. Destacamos el mayor tiempo de logueo en el grupo de CUOP (70 minutos) en relación con CUOB (40) (Fig. 2). No registramos diferencias entre los grupos en relación con los días de ingreso.
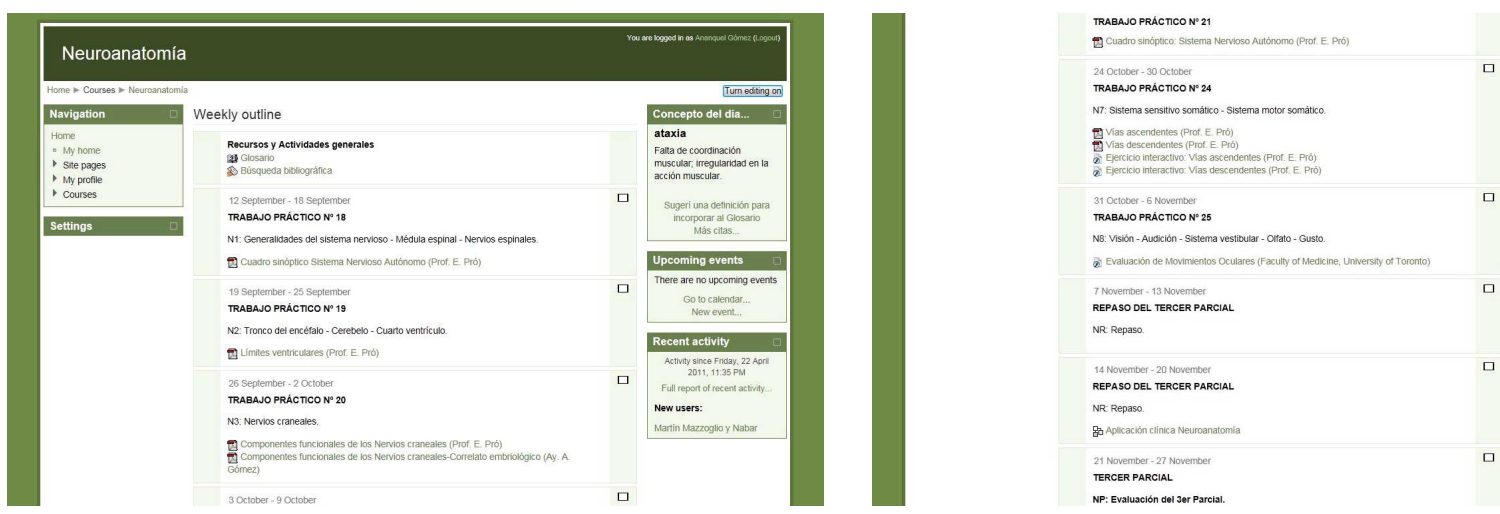

Fig. 1. Pantalla vista por los alumnos durante la rotación de Neuroanatomía realizada en Moodle®. 
En cuanto a la adherencia y beneficio subjetivo con el uso del EVEA registramos en ambos grupos una respuesta muy satisfactoria (promedio respuesta positiva $=$ CUOP 86,25\%; CUOB 87,78\%). Los resultados se exhiben en la Fig. 3.
Registramos una correlación significativa entre los resultados insatisfactorios en el examen y el menor uso del recurso (r=0,84; R2=0,78) (Fig.4) pero no registramos una correlación significativa con los puntajes de los exámenes aprobados (cantidad de horas logueado R2=0,32; cantidad de días de ingreso $\mathrm{R} 2=0,52$ ).

Tabla I. Porcentaje de respuestas sobre uso e ingreso al EVEA según comisión.

\begin{tabular}{|c|c|c|c|c|c|}
\hline \multirow{3}{*}{ Sexo } & \multicolumn{3}{|c|}{ CUOP 146} & \multicolumn{2}{|c|}{ CUOB 128} \\
\hline & Masculino & $37,67 \%$ & 55 & $31,25 \%$ & 40 \\
\hline & Femenino & $62,33 \%$ & 91 & $68,75 \%$ & 88 \\
\hline \multirow[t]{2}{*}{ Trabaja } & $\mathrm{Si}$ & $63,01 \%$ & 92 & $21,09 \%$ & 27 \\
\hline & No & $36,99 \%$ & 54 & $78,91 \%$ & 101 \\
\hline \multirow[t]{2}{*}{ ¿Tiene como alumno alguna experiencia previa de uso de e-learning? } & $\mathrm{Si}$ & $10,27 \%$ & 15 & $16,41 \%$ & 21 \\
\hline & No & $89,73 \%$ & 131 & $83,59 \%$ & 107 \\
\hline
\end{tabular}

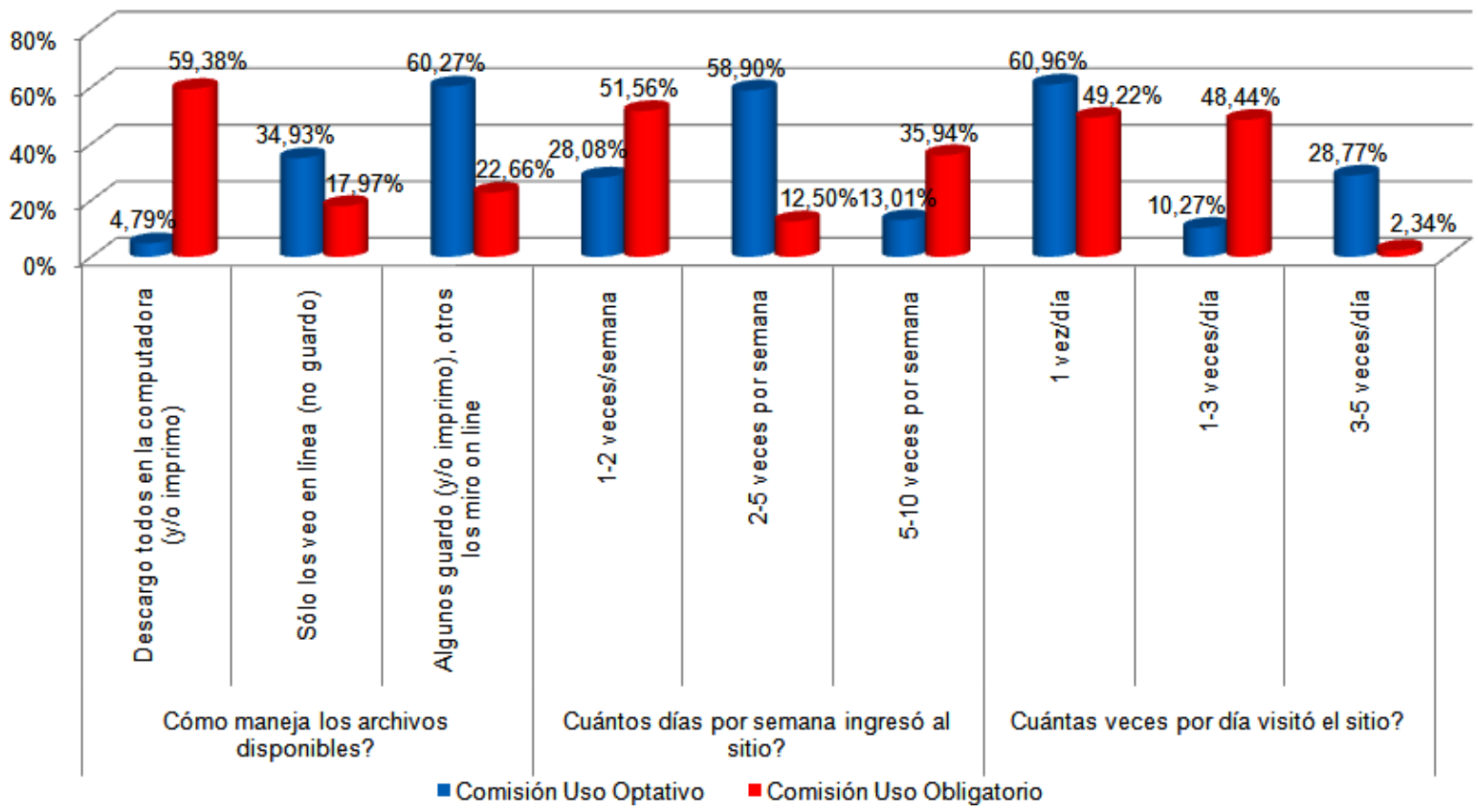

Fig. 2. Porcentaje de respuestas relacionadas con el lugar de ingreso, horarios y tiempo de logueo al EVEA según comisión.

\section{DISCUSIÓN}

La explosión de la Web y su injerencia social a finales de la década de los 90 puso de manifiesto la transformación de las nuevas tecnologías que generan los modos de acceder a la información y trabajar con ella en la construcción del conocimiento. No sólo las fuentes de información se ven transformadas, también las herramientas para interactuar con ellas y el sujeto que las utiliza. Diversas aplicaciones informáticas permiten manipular información en forma de texto, imágenes y sonidos, generando producciones nuevas con el influjo de la subjetividad de quien las 


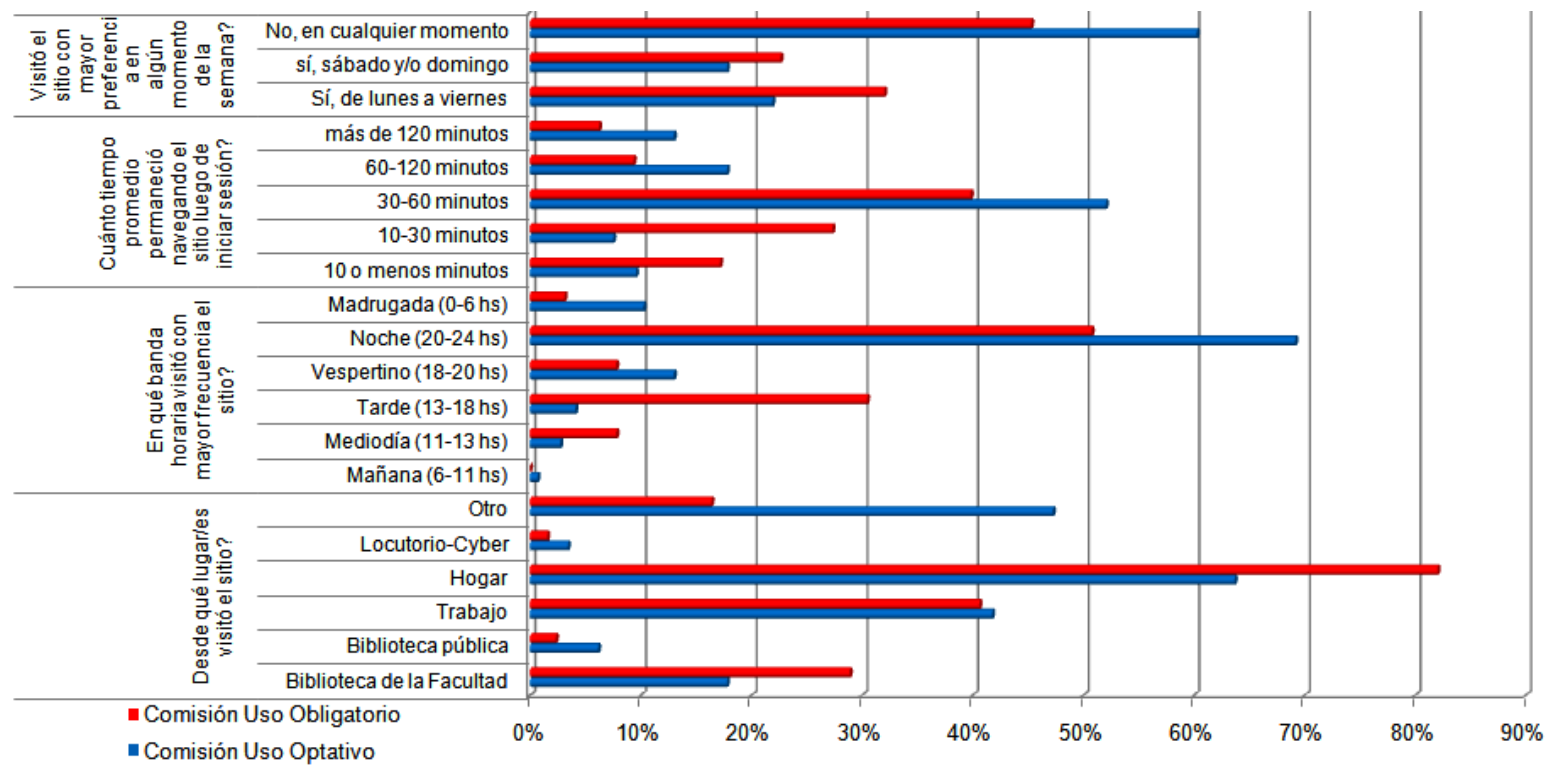

Fig. 3. Porcentaje de respuestas la adherencia y beneficios subjetivos obtenidos con el EVEA en ambas comisiones.

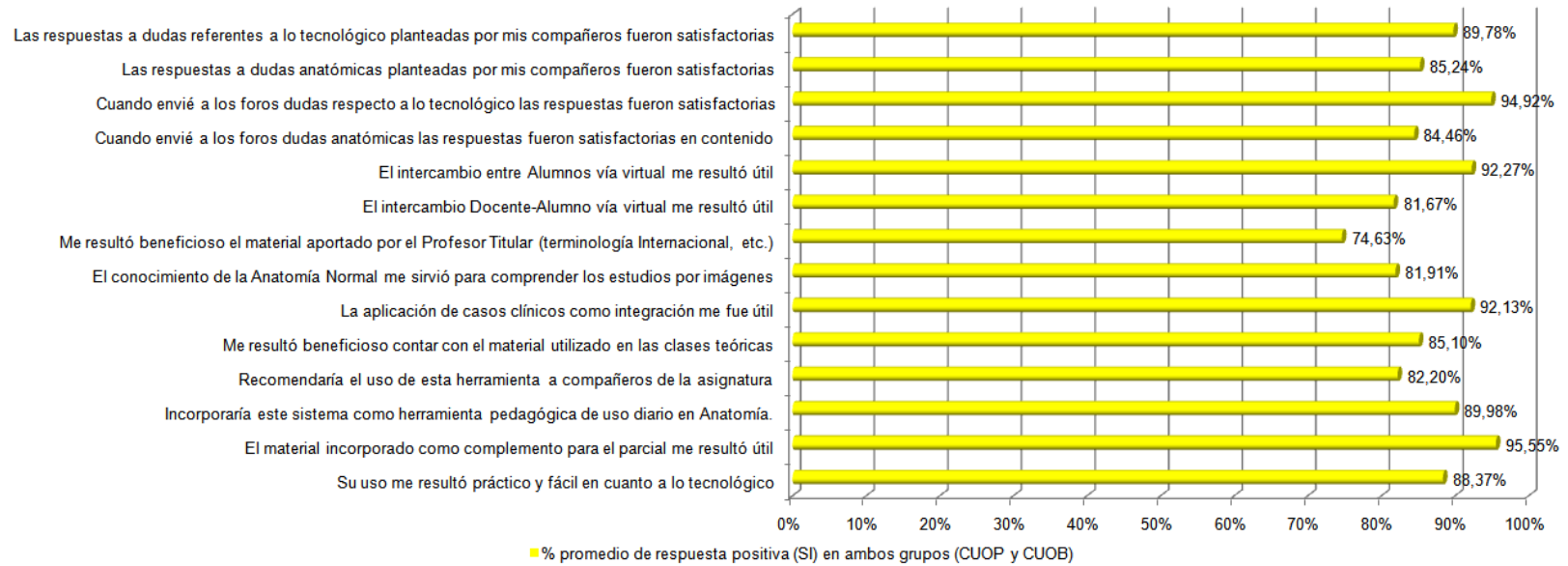

Fig. 4. Correlación entre el resultado insatisfactorio en el examen y uso del recurso.

modificó y reflejando su estructura cognitiva. Estas nuevas TICs transformaron los modos en que las comunidades académicas, científicas y profesionales trabajan. Y obligan a plantearnos cómo potenciarlas con el fin de acercar a los alumnos a comunidades académicas, científicas y profesionales que activamente producen conocimiento y lo publican on line.

Los cambios en las vías de comunicación generan oportunidades para imaginar nuevos escenarios educativos. En relación con la pedagogía, poder pensar nuevas tecnologías con el fin de apropiarse de ellas como herramienta para imaginar nuevos entornos de trabajo, nuevas actividades de aprendizaje y nuevas propuestas de enseñanza, siempre y cuando los avances sean en un marco de reflexión previo y continuo acerca de lo que hacemos, cómo lo hacemos y cómo podemos hacerlo.

Según Vigotsky, el conocimiento es un producto de la interacción social y la cultura, y sus procesos psicológicos superiores (comunicación, lenguaje, razonamiento y memoria) se adquieren primero en un contexto social y luego se internalizan; dicha internalización es producto del uso de un determinado comportamiento cognitivo en un contexto social. La importancia de la interacción social para el aprendizaje ha sido comprobada, un alumno aprende de forma más eficaz y significativa en un contexto de colaboración e intercambio con sus compañeros, existen mecanismos de carácter social que estimulan y favorecen el aprendizaje como las discusiones en grupo. Actualmente dicha comuni- 
TORNESE, E. B.; DOGLIOTTI, C. G.; MAZZOGLIO Y NABAR, M. J.; ALGIERI, R. D.; GAZZOTTI, A.; JIMÉNEZ VILLARRUEL, H. N.; REY, L. M. \& GÓMEZ, A. Entorno virtual de enseñanZa y aprendizaje aplicado como recurso instruccional complementario en la enseñanza de neuroanatomía: aspectos poblacionales, didácticos y psicopedagógicos. Int. J. Morphol., 29(4):1130-1135, 2011.

cación, colaboración y discusión traspasa los límites de lo real y se sumerge en la virtualidad donde los "jóvenes de la era digital" comparten y discuten muchos campos de su vida. Por tal motivo realizamos el EVEA en la plataforma Moodle (Modular Object Oriented Dynamic Learning Environment), fundada por Martin Dougiamas y basada en el constructivismo social (Burgos \& Koper, 2005; Correa Gorospe, 2005).

Un punto importante y fundamental para el docente y/o administradores del EVEA es conocer las representaciones que poseen los alumnos sobre lo que se les va a enseñar y analizar el proceso de interacción entre el conocimiento nuevo y el previo. Por tal motivo, en los TP y teóricos se abordó el tema previo a su trabajo en el EVEA, y se realizó un seguimiento individualizado de los resultados alcanzados por los participantes por sus ayudantes en los turnos y un seguimiento de los resultados a nivel macro por parte de los jefes de trabajos prácticos que coordinan el EVEA.

El uso de TICs influye en el conocimiento, percepciones y representaciones del mundo que tienen los usuarios con sus contactos reales y con otros que encuentran, cada vez más, mediados por tecnologías. Un alumno que tenga más oportunidades de aprender que otro, no sólo adquirirá más información sino que logrará un mayor desarrollo cognitivo. Más aún cuando dicha información ingresa por distintas vías sensoriales sincrónicas como en el caso del aprendizaje mediado por tecnologías. Es así como en el presente trabajo pudimos objetivar que, en la rotación de Neuroanatomía en que el objeto de estudio si bien real no es objetivable y las representaciones de los temas resultan abstractas e imprecisas para los educandos, los parámetros de ingreso, uso, adherencia y beneficio subjetivo con el EVEA como complementario de los TP fue muy satisfactorio en

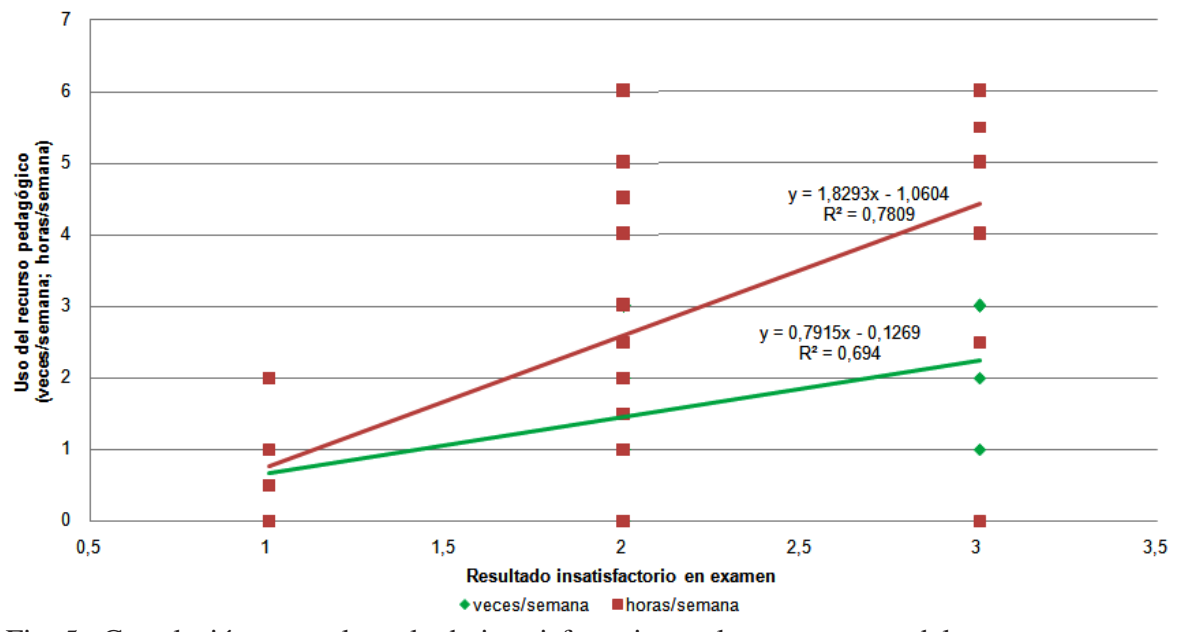

Fig. 5. Correlación entre el resultado insatisfactorio en el examen y uso del recurso. ambos grupos, destacándose una implicancia en los exámenes de aquéllos con un uso acotado del recurso instruccional.

Los EVEAs permiten recrear el "ambiente del aula" en forma virtual, generando espacios o comunidades organizadas en torno al aprendizaje, aportando soluciones globales para resolver aspectos relacionados con lo pedagógico. Para lograr este propósito cuentan con distintos tipos de herramientas: 1) herramientas para la comunicación (correo, foro, chat; aplicamos asincrónicas con docentes y sincrónicas entre participantes); 2) herramientas para administrar materiales (forma de subir y gestionar contenidos, es la parte activa y colaborativa); 3) herramientas para gestionar los estudiantes (permite seguimiento y evaluación de alumnos, muestran calificaciones, intervenciones y accesos) (Fig. 5).

Las plataformas están diseñadas desde una perspectiva constructivista y socio-cultural. El aprendizaje mediado por EVEAs supone afirmar que lo que el alumno aprende en el mismo no es simplemente una copia o reproducción de que en ese entorno se le presenta como contenido a aprender sino una reelaboración de ese contenido mediada por su estructura cognitiva. Por lo cual, la enseñanza en el EVEA tiene mucho que ver con la labor sincrónica entre profesores y alumnos, y su realización permite al alumno ir más allá de lo que podría hacer si estuviera solo con el material, acorde con lo expresado por los alumnos sobre la utilidad del intercambio.

Según Duart \& Sangrá (2000), frente a los EVEAs, existen 3 modelos distintos: modelo centrado en el estudiante, modelo centrado en los profesores y modelo centrado en los medios tecnológicos. En nuestro EVEA nos basamos en el modelo centrado en el estudiante que supone el auto-aprendizaje y la auto-transformación de esquemas cognitivos mediados por la tecnología disponible y el seguimiento individual del proceso de enseñanza y de aprendizaje por parte de tutores.

En conclusión, objetivamos beneficios en el empleo de EVEA por alumnos de anatomía en la rotación de neuroanatomía. Dicho recurso es una herramienta polivalente que genera la construcción del proceso de enseñanza y aprendizaje mediante la reelaboración del contenido mediada por la estructura cognitiva subjetiva del alumno. 
TORNESE, E. B.; DOGLIOTTI, C. G.; MAZZOGLIO Y NABAR, M. J.; ALGIERI, R. D.; GAZZOTTI, A.; JIMÉNEZ VILLARRUEL, H. N.; REY, L. M. \& GóMEZ, A. Entorno virtual de enseñanza y aprendizaje aplicado como recurso instruccional complementario en la enseñanza de neuroanatomía: aspectos poblacionales, didácticos y psicopedagógicos. Int. J. Morphol., 29(4):1130-1135, 2011.

TORNESE, E. B.; DOGLIOTTI, C. G.; MAZZOGLIO Y NABAR, M. J.; ALGIERI, R. D.; GAZZOTTI, A.; JIMÉNEZ VILLARRUEL, H. N.; REY, L. M. \& GÓMEZ, A. Virtual teaching and learning applied as supplementary instructional resources in teaching neuroanatomy: population, educational and psychopedagogic aspects. Int. J. Morphol., 29(4):1130-1135, 2011.

SUMMARY: New technologies of information and communication are changing society and strongly influence education, creating new scenarios valuable to the teaching and learning. These virtual teaching and learning (VLEs) are software platforms thought to facilitate pedagogical communication among participants in a context of educational process. The aim was to present results of adhesion, performance and educational value of the VLE, specifying underlying population concerns and educational psychology, when applied in neuroanatomy. We designed a VLE using Moodle ${ }^{\circledR}$ with consultation activities, homework, learning forums, wiki, hypertext and questionnaire. Implemented in 3 committees that submitted 2010 for 1 (obligatory) and 2 (not obligatory) and parameters were evaluated to know the effectiveness of the resource implemented as complementary educational curricula for practical work. The results were evaluated using descriptive statistical tools. $75 \%$ of students used the VLE, the frequency of increased access was at night (20-24 hours). We detected difficulties in the daily use of this tool despite its easy access. Half entered 2 times per week, without prevalence of that day, mostly one time per day from home and logged into the site, remaining between 30 and 70 minutes (60\%). We determined the correlation between unsatisfactory midterm exam performance and lower resource use (R2>0.78). We objectified benefit in the use of VLEs in the Neuroanatomy rotation. That resource generated the construction of teaching and learning by reworking the content mediated by the student subjective cognitive structure.

KEY WORDS: Neuroanatomy; Pedagogy; VLE.

\section{REFERENCIAS BIBLIOGRÁFICAS}

Algieri, R. D.; Ferrante, S. \& Mazzoglio y Nabar, M. J. Implementación de TIC en la enseñanza universitaria de la anatomía del hígado: aspectos neurobiológicos y psicopedagógicos, 2008. Disponible en: www.diegolevis.com.ar/secciones/Articulos/tic_medicina.pdf

Algieri, R. D.; Mazzoglio y Nabar, M. J.; Dogliotti, C. G. \& Gazzotti, A. TICs aplicadas a la enseñanza del aparato digestivo. Int. J. Morphol., 27(4):1261-8, 2009.

Burgos, D. \& Koper, R. Comunidades virtuales, grupos y proyectos de investigación sobre IMS Learning Design. Status quo, factores clave y retos inmediatos. RELIEVE, 11(2):189-200, 2005.

Correa Gorospe, J. M. La integración de plataformas de e-learning en la docencia universitaria: Enseñanza, aprendizaje e investigación con Moodle en la formación inicial del profesorado. RELATEC, 4(1):37-48, 2005.

Duart, J. M. \& Sangrá, A. Aprender en la virtualidad. Barcelona, Gedisa, 2000.

Litwin, E. Tecnologías educativas en tiempos de internet. $1^{\mathrm{a}}$ ed. Buenos Aires, Ed. Amorrortu, 2005.

Tornese, E. B.; Algieri, R. D.; Dogliotti, C. G.; Gazzotti, A.; Gómez, A.; Jiménez, H. N.; Mazzoglio y Nabar, M. J. \& Rey, L. Enseñanza audiovisual mediante TICs en Neurociencia. $12^{\circ} \mathrm{Jorna}-$ das Latinoamericanas de Neurociencia Cognitiva, Buenos Aires, 2010.
Dirección para correspondencia:

Martín J. Mazzoglio y Nabar, MD

Aráoz 1778 (C1414DQJ)

Ciudad Autónoma de Buenos Aires

ARGENTINA

Email: mazzoglioynabar@hotmail.com

Recibido : 02-05-2011

Aceptado: 22-07-2011 